\title{
Vibration analysis of rotor-bearing system using polynomial interpolation for squeeze film damper models
}

\author{
Tieshu Fan ${ }^{1}\left[\right.$ [ $\cdot$ Kamran Behdinan ${ }^{1}$
}

Received: 12 March 2020 / Accepted: 28 October 2020 / Published online: 4 December 2020

(c) Springer Nature Switzerland AG 2020

\begin{abstract}
The implementation of numerical squeeze film damper (SFD) models into rotordynamic study is a time-consuming work since it is challenging to efficiently calculate the SFD forces under a variety of different situations. This paper proposes a new scheme to incorporate SFD models into rotordynamic analysis. A polynomial interpolation technique is introduced to solve for SFD forces under different operating conditions. To evaluate the efficiency of the proposed method, a rotor shaft supported by an SFD at either end has been selected for vibration study, where a finite element model is created to describe the system. The transient response of the rotor at a steady state rotational speed is presented for an open SFD application. In addition, the frequency response is evaluated by the slow acceleration scheme. The prediction resulted from the interpolation method shows excellent agreement with that from the direct method. Moreover, the proposed method significantly reduces the simulation time for application using advanced SFD models.
\end{abstract}

Keywords Squeeze film damper · Rotor dynamics · Polynomial interpolation · Vibration analysis

\section{List of symbols}

C SFD film radial clearance

$D \quad$ SFD diameter

$D_{d, \text { in }}$

$D_{d, \text { out }}$

$D_{s, \text { in }}$

$D_{\text {s, out }}$

E

F

$F_{r} \quad$ SFD radial force

$F_{t} \quad$ SFD tangential force

$K_{b} \quad$ Bearing stiffness

$L \quad$ SFD length

$L_{s} \quad$ Shaft length

$m_{u} \quad$ Unbalanced mass

$p \quad$ Film pressure

$p_{a} \quad$ Ambient pressure

$p_{c} \quad$ Cavitation pressure

$p_{d} \quad$ Hydrodynamic pressure

$\begin{array}{ll}R & \text { SFD radius } \\ W_{d} & \text { Disk width } \\ z & \text { Axial direction } \\ \varepsilon & \text { Eccentricity ratio } \\ \theta & \text { Angular direction } \\ \mu & \text { Lubricant viscosity } \\ \rho_{d} & \text { Disk density } \\ \rho_{s} & \text { Shaft density } \\ \rho & \text { Lubricant density } \\ u & \text { Poisson's ratio } \\ \omega & \text { Whirling velocity } \\ \Delta t & \text { Time increment }\end{array}$

\section{Introduction}

Squeeze film dampers (SFDs) are lubricated bearing elements that are commonly applied in high-speed turbomachinery to provide mechanical support and dissipate

Tieshu Fan, tieshu@mie.utoronto.ca | 'Department of Mechanical and Industrial Engineering, University of Toronto, Toronto, ON, Canada. 
vibration energy [1]. The implementation of SFDs into aero engines limits the vibration amplitudes of the rotor at critical speeds and increases the onset of the rotor instability speed, both of which assist to solve the most commonly recurring vibration problems in rolling element bearings [2]. A comprehensive review of the relevant analytical and experimental work on SFDs has been conducted [1-4]. To incorporate SFD models in rotordynamic studies, three different approaches have been applied, namely, the rigid rotor model, the simple flexible rotor model, and the complex flexible rotor model.

In a rigid rotor model, the rotor shaft can be modeled using four degrees of freedom. A planar rotor carried in an SFD with linear centering spring was studied in [5], where the SFD was modeled using the short bearing approximation (SBA). To find the transient response and frequency response numerically, Runge Kutta integration technique was applied. Theoretical work has concluded that three steady-state orbit whirls may exist at the same frequency, which confirms the existence of a bi-stable condition and a jump phenomenon in rotordynamics [6]. The steady-state response of a centrally preloaded rigid rotor mounted on antifriction bearings supported by SFDs was obtained, where design parameters on SFDs were investigated to study the operating modes and force transmissibility [7]. For a rigid rotor supported by an eccentric SFD, the unbalanced response was approximated by a harmonic series, where the coefficients were determined by the collocation method and a nonlinear least-square regression [8]. The bifurcation analysis of a rigid rotor and SFD system with asymmetrical stiffness support were explored using analytical SFD forces from SBA [9].

In a simple flexible rotor model, the rotor mass is assumed to be either concentrated in the rotor center or distributed between the rotor center and the bearing center. The stability and synchronous steady-state operation of a centrally preloaded single mass flexible rotor was theoretically investigated, where the support SFDs were modeled using SBA $[10,11]$. The effectiveness of SFDs incorporated into a flexible rotor system without centralizing spring has been investigated analytically, where the rotor mass was lumped at the mid-point of a horizontal flexible shaft and at the bearing position [12]. The trigonometric collocation method has been used to determine the unbalanced response of a SFD-mounted flexible rotor, and the Floquet transition matrix method has been applied to evaluated solution stability [13]. The multi-solution response of simple flexible rotor supported on two identical SFDs with centralizing springs was studied by three methods including the synchronous circular centered-orbit (CCO) motion solution, the numerical integration method, and the slow acceleration method, where the SFD is modeled by the assumption of a short and cavitated oil film [14]. The numerical integration method has been also applied to analyze the design and operating parameters on the bifurcation of a simple flexible rotor, with and without centering springs on SFDs $[15,16]$.

In application of a complex flexible rotor model, the dynamic system usually includes unbalanced excitation from multiple masses or multiple shafts with multiple support bearings instead of a single mass on one shaft. In this case, the equation of system can be derived with multi-degrees of freedom. The stability of a radially symmetric multi-mass flexible rotor bearing system has been theoretically studied using linearized perturbation equations [17]. To find the synchronous unbalance response for general multi-degree of freedom rotor bearing systems, a set of nonlinear equations has been introduced [18]. An iterative method based on the secant root has been applied to determine the steady-state orbit whirls of multiple SFDs in a multishaft rotor system [19], where SFDs are modeled using short bearing or long bearing theory. A receptance harmonic balance method has been proposed for determining the periodic response of a complex rotorSFD system and the stability of the solution was verified by the Floquet Theory [20]. The influences of internal rotor material damping has been studied on a flexible rotor with two disks using the modal analysis [21].

Despite the tremendous effort in solving for the dynamic behavior of different rotor systems, the incorporated SFD models are usually simplified with either SBA or long bearing approximation (LBA), where some nonlinear thin film features such as fluid inertia, cavitation and lubricant leakages are not precisely modeled. Although some advanced SFD models have been developed to detect these nonlinear effects [22-26], challenges in simulating the frequency response of a rotordynamic system lie in efficiently obtaining the reaction forces from complicated SFD models, since the integration of numerical solutions from these models are time-consuming when SFD forces are needed under different eccentricities and speeds.

In applications with steady-state orbits, harmonic balance method and trigonometric collocation method have been employed to save the computational cost $[20,27]$. For numerical integration in the time domain to calculate a transient response, a linear interpolation scheme was applied to solve SFD forces using the existent forces from the closest points [28]. Chebyshev polynomials were also adopted to fit the SFD force and pressure [29, 30]. In this paper, a new polynomial interpolation scheme is proposed to incorporate the advanced SFD models.

This paper is organized as follows: first, the technique of SFD model interpolation is derived based on the squeezed film pressure and force. Subsequently, the developed method is applied on a rotor-SFD bearing model to evaluate its accuracy and efficiency in rotordynamic simulation, 
where the validated is carried out by an unbalanced-mass induced vibration of a one-shaft rotor suspended by two identical SFDs. Extensive discussions on the results are presented. Finally, the merits of the proposed scheme are concluded for further applications.

\section{SFD model interpolation}

The critical design consideration of an SFD is the amount of force it generates during operation, since too much force would make the system too rigid while too little force is
Since the Reynolds number is also linear to the operating speed, the hydrodynamic pressure in the squeezed film can be described by the following if the higher order term is neglected

$p_{d}(\varepsilon, \omega, \theta, z)=g(\varepsilon, \theta, z) \omega^{2}+h(\varepsilon, \theta, z) \omega$

where the first term on the right side of Eq. (5) represents the effect of fluid inertia and the second term represents the effect of viscous flow.

Substituting Eq. (5) into Eq. (1) and integrating it over the film domain yield the reaction force, i.e.

$F(\varepsilon, \omega)=\left[\begin{array}{l}F_{r}(\varepsilon, \omega) \\ F_{t}(\varepsilon, \omega)\end{array}\right]=\int_{0}^{L} \int_{0}^{2 \pi} p(\varepsilon, \omega, \theta, z)\left[\begin{array}{c}\cos \theta \\ \sin \theta\end{array}\right] d \theta d z=\left[\begin{array}{l}G_{r}(\varepsilon) \\ G_{t}(\varepsilon)\end{array}\right] \omega^{2}+\left[\begin{array}{l}H_{r}(\varepsilon) \\ H_{t}(\varepsilon)\end{array}\right] \omega+\left[\begin{array}{l}I_{r}(\varepsilon) \\ I_{t}(\varepsilon)\end{array}\right]$

insufficient to reduce the vibration amplitudes. In theory, SFD forces are calculated by integrating the hydrodynamic pressure in the squeezed film. If the circular-centered orbit motion is assumed for the bearing in a symmetric system, the pressure within of the squeezed film can be determined by the operating speed and the whirling eccentricity ratio. If the pressure is further assumed to remain constant in the region when cavitation occurs, the following equation can be derived to describe the film pressure in SFD:

$p(\varepsilon, \omega, \theta, z)= \begin{cases}p_{d}(\varepsilon, \omega, \theta, z)+p_{a} & \text { in full }- \text { film region } \\ p_{c} & \text { in cavitation region }\end{cases}$

where $p_{a}$ and $p_{c}$ refer to the ambient pressure and the cavitation pressure for the applied lubricant, respectively. $p_{d}$ represents the hydrodynamic pressure that is generated by the squeezed motion of the journal bearing.

According to the thin film theory, the hydrodynamic pressure can be solved by the momentum equation and the continuity equation [31]. For the operation under small Reynolds number, the pressure can be approximated by the perturbation method as follows [32].

$p_{d}=p_{0}+\operatorname{Re} p_{1}+O\left(\operatorname{Re}^{2}\right)$

where $p_{0}$ represents to the purely viscous pressure, $p_{1}$ is the first-order inertia pressure, and $O\left(\mathrm{Re}^{2}\right)$ is the inertia correction that is assumed to be of order $\mathrm{Re}^{2}$. This approximation is strictly valid for small values of Reynolds number, i.e. $\operatorname{Re} \ll 1$.

The purely viscous pressure $p_{0}$ and the first-order inertia pressure $p_{1}$ are linear to the operating speed [33], i.e.

$p_{0} \propto \omega$

$p_{1} \propto \omega$ where $G_{i}$ and $H_{i}(i=r, t)$ are results integrated from $g(\varepsilon, \theta, z)$ and $h(\varepsilon, \theta, z)$ in Eq. (2), respectively. Both $G$ and $H$ are nonlinear functions of the eccentricity ratio $\varepsilon . l_{j}(i=r, t)$ is an integration constant, which can be determined by the pressure and the extent of the cavitation.

Equation (6) can be written as follows in vector forms

$F(\varepsilon, \omega)=\left[\begin{array}{lll}G & H & l\end{array}\right]\left[\begin{array}{lll}\omega^{2} & \omega & 1\end{array}\right]^{T}$

where $G=\left[\begin{array}{l}G_{r}(\varepsilon) \\ G_{t}(\varepsilon)\end{array}\right], H=\left[\begin{array}{l}H_{r}(\varepsilon) \\ H_{t}(\varepsilon)\end{array}\right], I=\left[\begin{array}{c}I_{r}(\varepsilon) \\ I_{t}(\varepsilon)\end{array}\right]$.

Assuming that the nonlinear functions $G, H$ and $/$ can be further approximated by polynomials, i.e.

$$
\begin{aligned}
& G(\varepsilon)=G_{0}+G_{1} \varepsilon+G_{2} \varepsilon^{2}+\cdots+G_{n} \varepsilon^{n} \\
& H(\varepsilon)=H_{0}+H_{1} \varepsilon+H_{2} \varepsilon^{2}+\cdots+H_{n} \varepsilon^{n}
\end{aligned}
$$

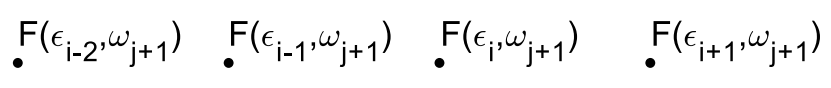

$$
\begin{aligned}
& \text {. }\left(\epsilon_{\mathrm{i}-2}, \omega_{\mathrm{j}}\right) \quad \text {. } \mathrm{F}\left(\epsilon_{\mathrm{i}-1}, \omega_{\mathrm{j}}\right) \quad \text {. } \mathrm{F}\left(\epsilon_{\mathrm{i}}, \omega_{\mathrm{j}}\right) \quad \text {. }\left(\epsilon_{\mathrm{i}+1}, \omega_{\mathrm{j}}\right) \\
& F(\epsilon, \omega)
\end{aligned}
$$

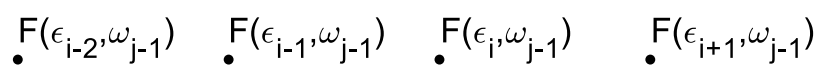

Fig. 1 Local indexing for solving $F(\varepsilon, \omega)$ by neighboring forces 
$I(\varepsilon)=I_{0}+I_{1} \varepsilon+I_{2} \varepsilon^{2}+\cdots+I_{n} \varepsilon^{n}$

where $G_{i}, H_{i} I_{i}(i=1,2, \cdots, n)$ refer to two-dimentional vectors that include only constants.

Equation (7) can be re-written as

$F(\varepsilon, \omega)=\left[\begin{array}{lllll}1 & \varepsilon & \varepsilon^{2} & \cdots & \varepsilon^{n}\end{array}\right][C]\left[\begin{array}{lll}\omega^{2} & \omega & 1\end{array}\right]^{T}$

where

$[C]=\left[\begin{array}{ccc}G_{0} & H_{0} & I_{0} \\ G_{1} & H_{1} & I_{1} \\ G_{2} & H_{2} & I_{2} \\ \vdots & \vdots & \vdots \\ G_{n} & H_{n} & I_{n}\end{array}\right]$

All matrix entries in Eq. (12) can be solved together via pre-calculating the forces from an SFD model. To be specific, a series of neighboring forces at different operating conditions (i.e. at certain eccentricity ratios and whirling speeds), as shown in Fig. 1, are first calculated from an SFD model, where $F\left(\varepsilon_{i}, \omega_{j}\right)$ is defined as the pre-calculated force under which the eccentricity ratio and the whirling speed are higher than but close to the unknown force $F(\varepsilon, \omega)$. Accordingly, the matrix [C]can be expressed as follows

$[C]=[E]^{-1}[F][W]^{-1}$

where
$[E]=\left[\begin{array}{ccccc}1 & \varepsilon_{i-\left[\frac{n}{2}\right]} & \varepsilon_{i-\left[\frac{n}{2}\right]}^{2} & \cdots & \varepsilon_{i-\left[\frac{n}{2}\right]}^{n} \\ 1 & \varepsilon_{i-\left[\frac{n}{2}\right]+1} & \varepsilon_{i-\left[\frac{n}{2}\right]+1}^{2} & \cdots & \varepsilon_{i-\left[\frac{n}{2}\right]+1}^{n} \\ 1 & \varepsilon_{i-\left[\frac{n}{2}\right]+2} & \varepsilon^{2}-\left[\frac{n}{2}\right]+2 & \cdots & \varepsilon^{n}\left[-\left[\frac{n}{2}\right]+2\right. \\ \vdots & \vdots & \vdots & \ddots & \vdots \\ 1 & \varepsilon_{i-\left[\frac{n}{2}\right]+n} & \varepsilon_{i-\left[\frac{n}{2}\right]+n}^{2} & \cdots & \varepsilon^{n}-\left[\frac{n}{2}\right]+n\end{array}\right]$

$[F]=\left[\begin{array}{ccc}F\left(\varepsilon_{i-\left[\frac{n}{2}\right]}, \omega_{j-1}\right) & F\left(\varepsilon_{i-\left[\frac{n}{2}\right]^{\prime}}, \omega_{j}\right) & F\left(\varepsilon_{i-\left[\frac{n}{2}\right]}, \omega_{j+1}\right) \\ F\left(\varepsilon_{i-\left[\frac{n}{2}\right]+1^{\prime}}, \omega_{j-1}\right) & F\left(\varepsilon_{i-\left[\frac{n}{2}\right]+1^{\prime}} \omega_{j}\right) & F\left(\varepsilon_{i-\left[\frac{n}{2}\right]+1^{\prime}} \omega_{j}\right) \\ F\left(\varepsilon_{i-\left[\frac{n}{2}\right]+2^{\prime}}, \omega_{j-1}\right) & F\left(\varepsilon_{i-\left[\frac{n}{2}\right]+2^{\prime}} \omega_{j}\right) & F\left(\varepsilon_{i-\left[\frac{n}{2}\right]+2^{\prime}} \omega_{j}\right) \\ \vdots & \vdots & \vdots \\ F\left(\varepsilon_{i-\left[\frac{n}{2}\right]+n^{\prime}}, \omega_{j-1}\right) & F\left(\varepsilon_{i-\left[\frac{n}{2}\right]+n^{\prime}} \omega_{j}\right) & F\left(\varepsilon_{i-\left[\frac{n}{2}\right]+n^{\prime}} \omega_{j}\right)\end{array}\right]$

$[W]=\left[\begin{array}{lll}\omega_{j-1}^{2} & \omega_{j}^{2} & \omega_{j+1}^{2} \\ \omega_{j-1} & \omega_{j} & \omega_{j+1} \\ 1 & 1 & 1\end{array}\right]$

Since the order of the polynomials can be any positive integer, the subscript $\left[\frac{n}{2}\right]$ is introduced as the smallest integer equal or greater than $\frac{n}{2}$ such that the unknown force is weighted evenly by its neighboring forces.

Substitution of Eq. (13) into Eq. (11) implies that the unknown force $F(\varepsilon, \omega)$ can be associated with a series of known forces with an ordered set of indices $(i, j)$, where
Fig. 2 Algorithm for rotordynamic analysis with SFD interpolation
Generate a map of $F\left(\varepsilon_{i}, \omega_{j}\right)$

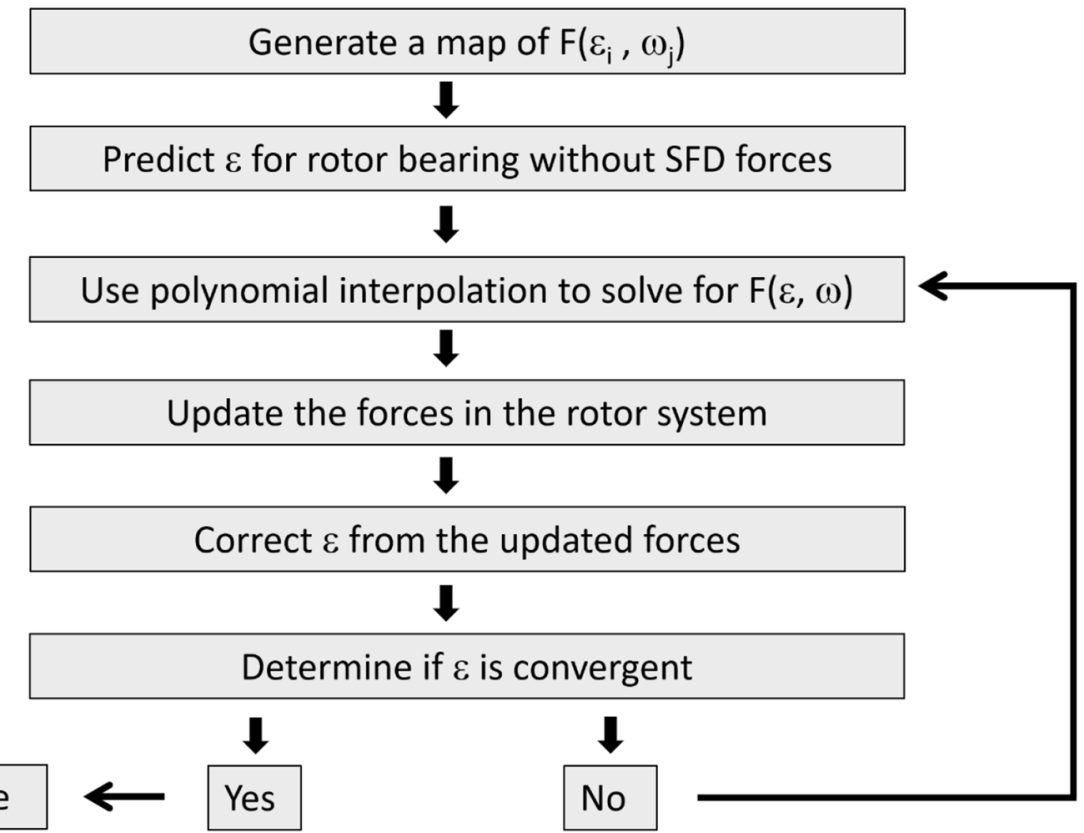




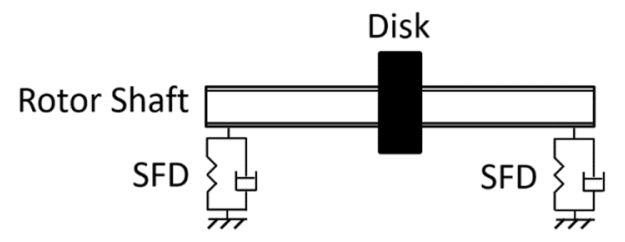

Fig. 3 Rotor system schematic

Table 1 Simulation parameters for rotor-SFD system

\begin{tabular}{lll}
\hline Parameter & Value & Unit \\
\hline$c / R$ & 0.00485 & \\
$D_{d, \text { in }}$ & 0.0603 & $\mathrm{~m}$ \\
$D_{d, \text { out }}$ & 0.2032 & $\mathrm{~m}$ \\
$D_{s, \text { in }}$ & 0.0413 & $\mathrm{~m}$ \\
$D_{s, \text { out }}$ & 0.0603 & $\mathrm{~m}$ \\
$E$ & 211 & $\mathrm{GPa}$ \\
$K_{b}$ & 17.5 & $\mathrm{~kg} / \mathrm{mm}$ \\
$L_{s}$ & 0.5994 & $\mathrm{~m}$ \\
$L / D$ & 0.325 & \\
$m_{u}$ & 0.00036 & $\mathrm{~kg} \cdot \mathrm{m}$ \\
$W_{d}$ & 0.0508 & $\mathrm{~m}$ \\
$\mu$ & 0.0227 & $\mathrm{~Pa} \cdot \mathrm{s}$ \\
$\rho_{d}$ & 7810 & $\mathrm{~kg} / \mathrm{m}^{3}$ \\
$\rho_{s}$ & 7810 & $\mathrm{~kg} / \mathrm{m}^{3}$ \\
$\rho$ & 1003.5 & $\mathrm{~kg} / \mathrm{m}^{3}$ \\
$U$ & 0.29 & \\
\hline
\end{tabular}

each index varies over a fixed range, independent of the values of the other index. Neighboring forces have associated indices that differ by plus or minus one.
In other words, if a map of SFD forces are pre-calculated at respective eccentricity ratios and whirling speeds, forces at other eccentricity ratios and whirling speeds can be solved by the polynomial interpolation without going through the SFD model. This technique is extremely beneficial in rotordynamic simulation when SFD forces are required under thousands of different conditions.

Figure 2 shows a diagram of the interpolation algorithm in a rotordynamic application, where the predictor-corrector technique is incorporated during iteration. In the following case studies, the predictor uses backward finite difference method and the corrector uses Newmark-Beta method.

\section{Application and results}

A Jeffcott rotor supported by two identical SFDs at either end, as shown in Fig. 3 , is selected to access the efficiency of the proposed technique at high operating speeds. The rotor vibration is excited by an unbalanced mass on the central disk. SFDs are executed without seals to stop the oil leakage. Instead of using the conventional short bearing model, a finite length bearing model is applied to calculate the SFD forces with more accuracy. The dimensions of the rotor shaft and SFDs as well as lubricant properties are listed in Table 1.

Two scenarios are studied: (i) the frequency response of the rotor, which is solved by the slow acceleration method; (ii) the transient response of the rotor, which is found by a steady-state study. Results are compared between the polynomial interpolation and the conventional method
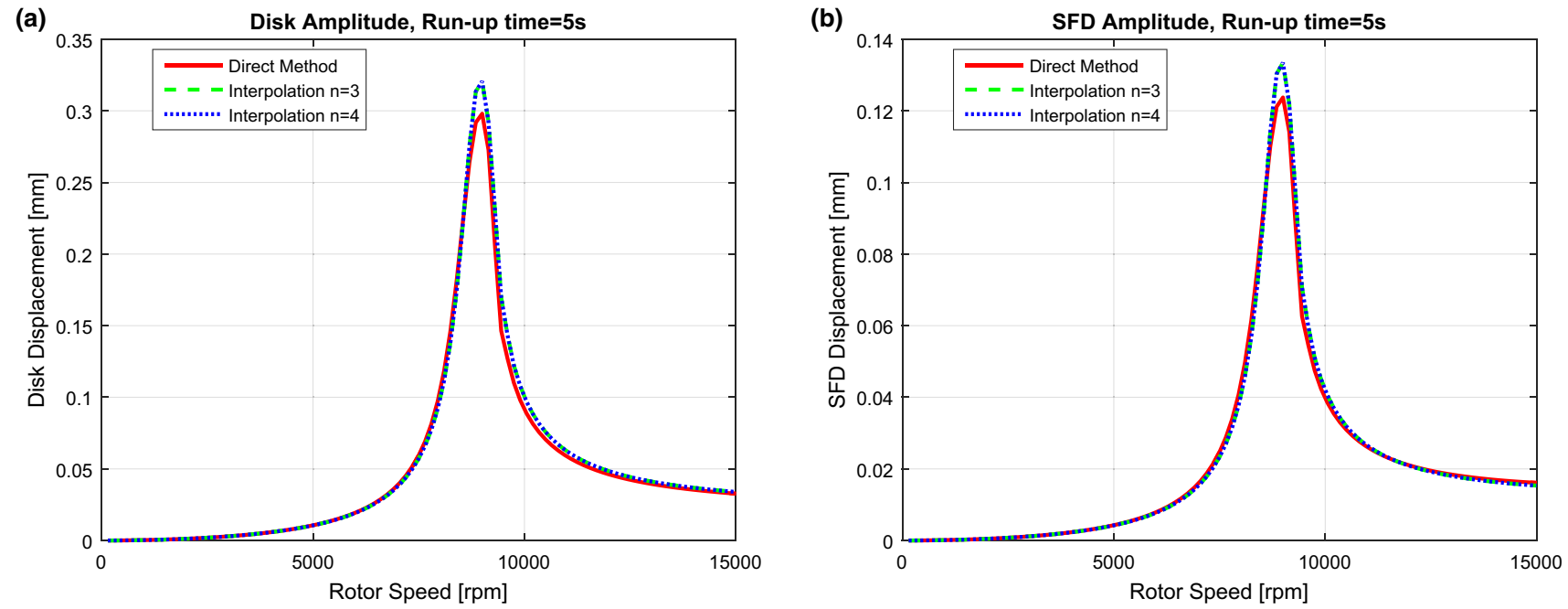

Fig. 4 Frequency response function (a) disk displacement (b) SFD displacement 

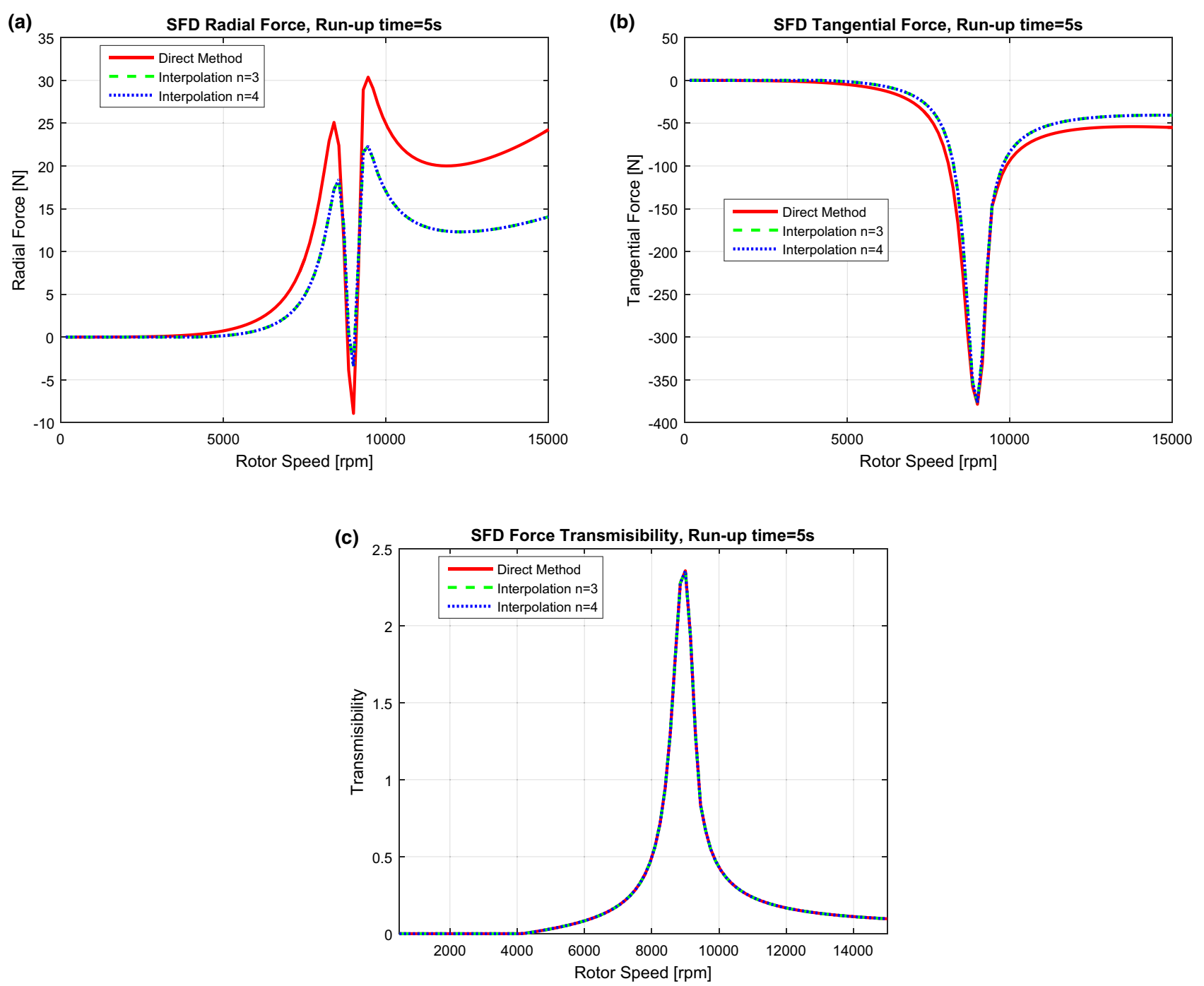

Fig. 5 SFD force over speed range of 0-15,000 RPM (a) Radial force (b) Tangential force (c) Force transmissibility

(where SFD forces are directly calculated from the SFD model in each iteration). In the polynomial interpolation, the order of polynomial approximation is studied in an odd number and an even number respectively to represent the robustness of the algorithm. During the process of creating an SFD force map for different operating conditions, the increment of the eccentricity ratio is 0.01 and the increment of the whirling speed is $1000 \mathrm{rpm}$.

Figure 4 shows the frequency response function of the system solved by the slow acceleration method when the rotor passes the first critical speed. Figure $4 \mathrm{a}$ is the disk amplitude, where the spike shows the critical speed is around $9000 \mathrm{rpm}$. The displacement predicted by the polynomial interpolation matches well with the direct method, except for the range that is close to the critical speed, where the interpolation method slightly over-predicted the amplitude for $8 \%$. Figure $4 \mathrm{~b}$ is the vibration amplitude at the SFD end. Compared to the disk amplitude, the SFD amplitude is much lower. The difference between the displacements at different locations can be further utilized to determine the mode shape of the rotor. The interpolation method gives the amplitude slightly higher than the direct method only at the critical speed though the critical speed is the same.

Figure 5 displays the SFD reaction force over the same speed range. Figure $5 \mathrm{a}$ is the radial force, where a sudden

Table 2 Simulation time for the slow acceleration method

\begin{tabular}{lllll}
\hline & Direct method & \multicolumn{2}{l}{$\begin{array}{l}\text { Polynomial interpola- } \\
\text { tion }\end{array}$} \\
\cline { 2 - 5 } & & $n=2$ & $n=3$ & $n=4$ \\
\hline CPU cost (in second) & 65,250 & 1500 & 1501 & 1500 \\
\hline
\end{tabular}


sink has been recorded when the speed reaches to the critical speed. This result occurs due to the presence of fluid inertia in the oil film, whose effect is significant when the journal eccentricity ratio becomes high and it could possibly change the direction of radial force from positive to negative. The same result has been previously reported in the literature [34]. The interpolation method underestimates the radial force for $25 \%$ around the critical speed, which leads to a higher displacement prediction as shown in Fig. 4. Figure $5 \mathrm{~b}$ plot the tangential force, where an excellent agreement has been shown between the interpolation method and the direct method. The dramatic increase of the tangential force at the critical speed proves the damping competency of the SFD in attenuating the rotor vibration. Figure $5 c$ is the force transmissibility of the SFD. Since the tangential force is one order larger than the radial force, the force transmissibility is primarily determined by the tangential force.

Both Figs. 4 and 5 show that the difference is negligible between the interpolation methods considering different orders of polynomial series. This is a result of the high resolution of the force map that it generates during the precalculation stage. Since the neighboring forces in the force map has close eccentricity ratio and operating speed, the interpolation results would show little difference under different orders. The small difference between the direct method and interpolation method also verifies the reliability and stability of the proposed polynomial approximation.

Table 2 displays the computational cost for the simulation models using an ' 8 i7-core $3.40 \mathrm{GHz}$ ' CPU, where a significant cost reduction has been shown by the polynomial interpolation. In this slow acceleration scheme, the rotor speed is increased from 0 to $15,000 \mathrm{rpm}$ in $5 \mathrm{~s}$ with a time-step of 1 microsecond. In other words, it has 5 million time-steps with different SFD conditions in each time step. If the direct method is applied to simulate the rotor vibration, the SFD model must be solved 5 million times, which is the reason that it slows down the simulation. By contrast, the polynomial interpolation only requires the SFD forces to be calculated a limited number of times. Based on the same resolution of the force map as discussed previously, the SFD model is called 1500 times, which is three orders less than that of the direct method. In addition, the same amount of times is needed for different orders of polynomial approximation since the interpolation takes little time.
Figure 6 presents the transient orbit at the critical speed from a steady-state simulation. Figure $6 a, c$ and e are results generated by the direct method, while the Fig. $6 b, d$ and $f$ are results predicted by the polynomial interpolation. Figure 6a-d show that the motion of central disk and SFD are almost identical between these two methods, expect for the amplitude where the polynomial method gently provides higher displacement for $10 \%$. The orbit is circular centered since the system has a symmetric structure. Figure $6 \mathrm{e}$ and $\mathrm{f}$ show that the whirling eccentricity from the interpolation method is slightly higher than that from the direct method for $10 \%$, which is the result of the lowered force prediction at high eccentricities.

\section{Conclusion}

This work introduces a polynomial interpolation technique for SFD model applications. It benefits the rotordynamic simulation by reducing the computational cost and makes any sophisticated SFD model readily available for transient study. The proposed method requires the SFD model calculation be performed only a limited number of times based on a selected set of eccentricity ratios and whirling speeds. Instead of going through the numerical computation of the SFD model for every changed condition in the rotordynamic simulation, the polynomial interpolation can be introduced in every time step to quickly estimate the SFD forces.

A symmetric rotor with a support system consisting of two identical SFDs is studied to evaluate the efficiency of the polynomial interpolation. Both the frequency response function and the steady state operation are studied to show the stability and reliability of the proposed method. Results show that the new method predicts excellent rotor behavior compared to the direct method while significantly reducing the simulation time.

By selecting a proper order and the increments in the eccentricity and the speed while applying the polynomial interpolation in SFD models, rotordynamic analysis can be achieved more efficiently and effectively. Other complicated SFD models could be analyzed in the future via using this method to explore the SFD effect in highspeed turbomachinery. 


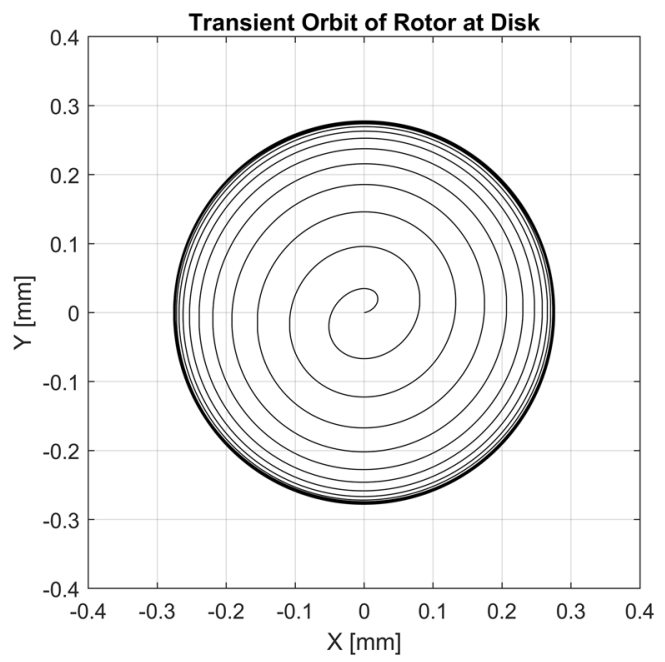

(a)

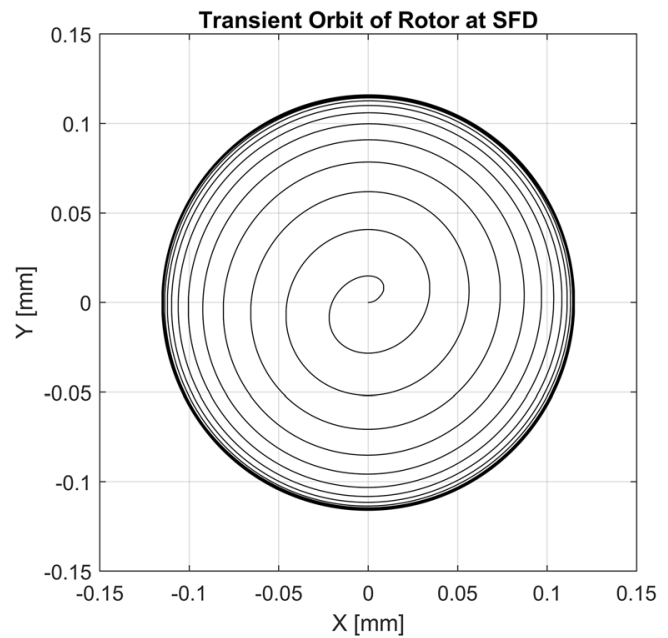

(c)

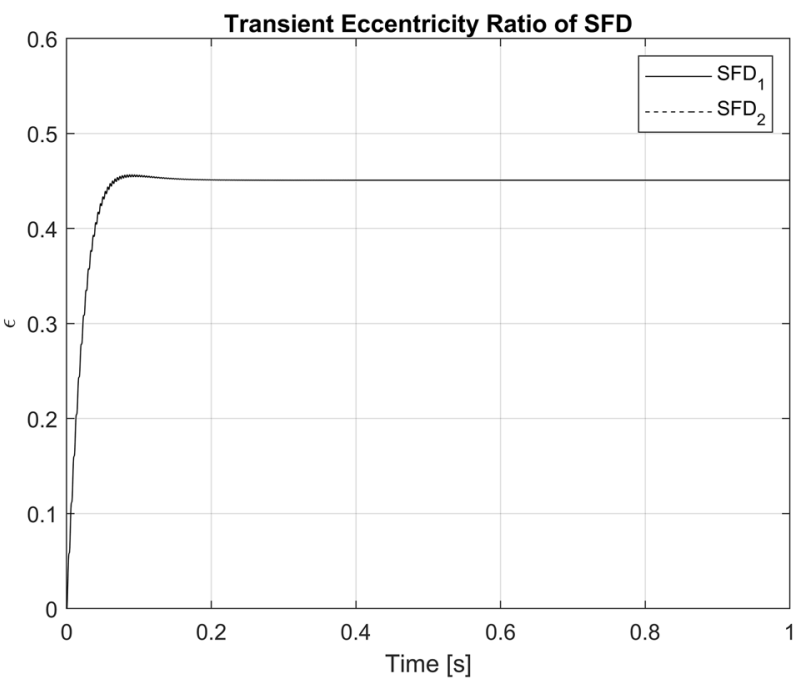

(e)

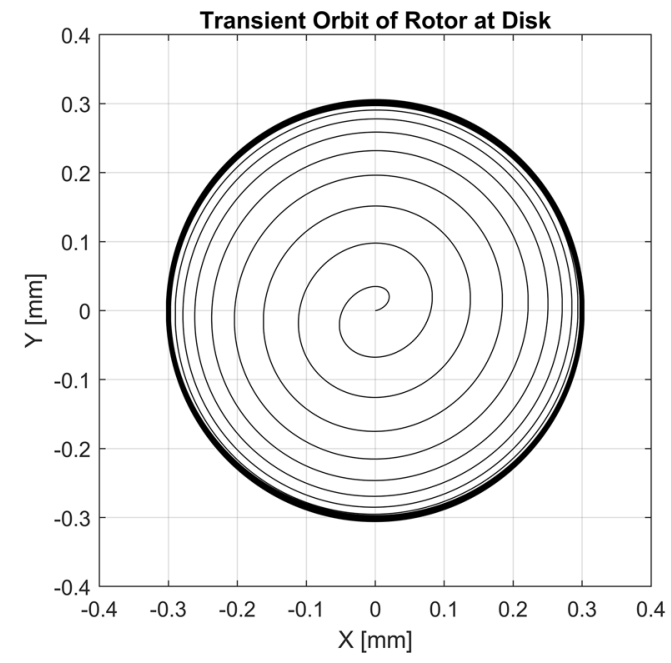

(b)

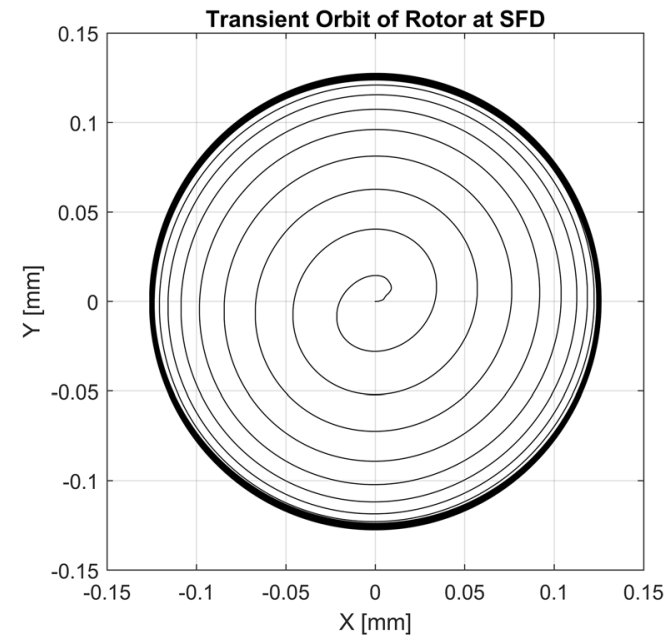

(d)

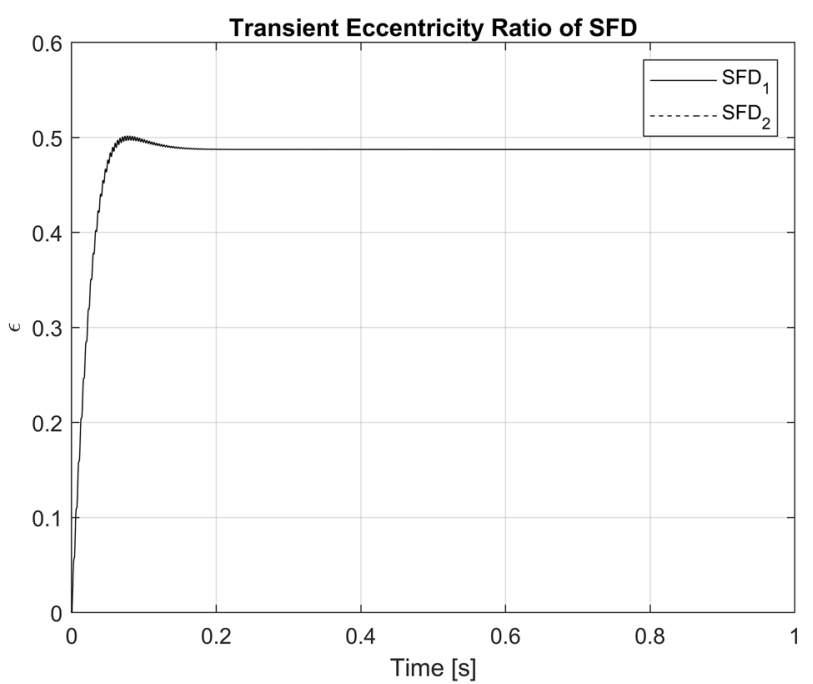

(f) 
४Fig. 6 Transient displacement of rotor-SFD system at 9000 RPM (a) Disk orbit by the direct method (b) Disk orbit by the polynomial interpolation (c) SFD orbit by the direct method (d) SFD orbit by the polynomial interpolation (e) Eccentricity ratio by the direct method (f) Eccentricity ratio by the polynomial interpolation

Acknowledgements This work is supported by Natural Sciences and Engineering Research Council of Canada (NSERC), Pratt \& Whitney Canada, and Mitacs Canada.

\section{Compliance with ethical standards}

Conflict of interest The authors declare that they have no conflict of interest.

\section{References}

1. Zeidan FY, San Andres L, Vance JM (1996) Design and application of squeeze film dampers in rotating machinery. Proc twenty-fifth Turbomach Symp 20

2. Pietra LD, Adiletta G (2002) The squeeze film damper over four decades of investigations. Part I: characteristics and operating features. Shock Vib Dig 34:3-26

3. Adiletta G, Pietra LD (2002) The squeeze film damper over four decades of investigations. Part II: Rotordynamic analyses with rigid and flexible rotors. Shock Vib Dig 34:97-126

4. San Andrés L, Jeung S, Den S, Savela G (2016) Squeeze film performance dampers: experimental appraisal of their dynamic performance. Proc 2016 Asia Turbomach Pump Symp pp 1-23

5. Taylor DL, Kumar BRK (1981) Closed-form, steady-state solution for the unbalance response of a rigid rotor in squeeze film damper. J Eng Power 105:551-556

6. White DC (1972) The dynamics of a rigid rotor supported on squeeze film bearings. In: Conference on vibration in rotating systems. London, England, pp 213-229

7. Mohan S, Hahn EJ (1974) Design of squeeze film damper supports for rigid rotors. J Eng Ind 96:976-982

8. Zhao JY, Linnett IW, McLean LJ (1994) Subharmonic and quasi-periodic motions of an eccentric squeeze film dampermounted rigid rotor. J Vib Acoust 116:357-363

9. Chen H, Hou L, Chen Y (2017) Bifurcation analysis of a rigidrotor squeeze film damper system with unsymmetrical stiffness supports. Arch Appl Mech 87:1347-1364

10. Rabinowitz MD, Hahn EJ (1977) Stability of squeeze-filmdamper supported flexible rotors. J Eng Power 99:545-551

11. Rabinowitz MD, Hahn EJ (1977) Steady-state performance of squeeze film damper supported flexible rotors. J Eng Power 99:552-558

12. Cookson RA, Kossa SS (1980) The effectiveness of squeeze-film damper bearings supporting flexible rotors without a centralising spring. Int J Mech Sci 22:313-324

13. Zhao JY, Linnett IW, McLean LJ (1994) Stability and bifurcation of unbalanced response of a squeeze film damped flexible rotor. J Tribol 116:361-368

14. Zhu CS, Robb DA, Ewins DJ (2002) Analysis of the multiplesolution response of a flexible rotor supported on non-linear squeeze film dampers. J Sound Vib 252:389-408. https://doi. org/10.1006/jsvi.2001.3910

15. Inayat-Hussain ال I (2005) Bifurcations of a flexible rotor response in squeeze-film dampers without centering springs. Chaos Solitons Fractals 24:583-596. https://doi.org/10.1016/j.chaos .2004.09.047

16. Inayat-Hussain $\mathrm{Jl}$ (2009) Bifurcations in the response of a flexible rotor in squeeze-film dampers with retainer springs. Chaos Solitons Fractals 39:519-532. https://doi.org/10.1016/j.chaos .2007.01.086

17. McLean LJ, Hahn EJ (1985) Stability of squeeze film damped multi-mass flexible rotor bearing systems. J Tribol 107:402-409

18. McLean LJ, Hahn EJ (1983) Unbalance behavior of squeeze film damped multi-mass flexible rotor bearing systems. J Lubr Technol 105:22-28

19. Greenhill LM, Nelson HD (1982) Iterative determination of squeeze film damper eccentricity for flexible rotor systems. J Mech Des 104:334-338

20. Bonello P, Brennan MJ (2004) A study of the nonlinear interaction between an eccentric squeeze film damper and an. J Eng Gas Turbines Power 126:855-866. https://doi.org/10.1115/1.17875 03

21. Chouksey M, Dutt JK, Modak S V. (2012) Modal analysis of rotorshaft system under the influence of rotor-shaft material damping and fluid film forces. In: Mechanism and machine theory

22. Fan T, Hamzehlouia S, Behdinan K (2017) The effect of lubricant inertia on fluid cavitation for high-speed squeeze film dampers. J Vibroeng 19:6122-6134. https://doi.org/10.21595/ jve.2017.19314

23. Fan T, Behdinan K (2017) The evaluation of linear complementarity problem method in modeling the fluid cavitation for squeeze film damper with off-centered whirling motion. Lubricants 5:46. https://doi.org/10.3390/lubricants5040046

24. Fan T, Behdinan K (2019) Investigation into the effect of piston ring seals on an integrated squeeze film damper model. J Mech Sci Technol 33:559-569. https://doi.org/10.1007/s1220 6-019-0109-4

25. Fan T, Behdinan $\mathrm{K}$ (2018) The evaluation of modelling techniques for lubricant cavitaion in the application of squeeze film dampers. In: CSME International Congress 2018. p 33

26. Andres LS, Jeung SH (2016) Orbit-model force coefficients for fluid film bearings: a step beyond linearization. J Eng Gas Turbines Power 138:022502. https://doi.org/10.1115/1.4031237

27. Chen C, Natsiavas S, Nelson H (1998) Coupled lateral-torsional vibration of a gear-pair system supported by a squeeze film damper. J Vib Acoust ASME 120:860-867

28. Chen Z, Jiao Y, Xia S et al (2002) An efficient calculation method of nonlinear fluid film forces in journal bearing. Tribol Trans 45:324-329. https://doi.org/10.1080/10402000208982556

29. Rodrigues FA, Thouverez F, Gibert C, Jezequel L (2003) Chebyshev polynomials fits for efficient analysis of finite length squeeze film damped rotors. J Eng Gas Turbines Power 125:175183. https://doi.org/10.1115/1.1423319

30. Groves $\mathrm{KH}$, Bonello P (2010) Improved identification of squeezefilm damper models for aeroengine vibration analysis. Tribol Int 43:1639-1649. https://doi.org/10.1016/j.triboint.2010.03.010

31. Szeri AZ (1999) Fluid film lubrication: theory and design. Cambridge University Press, Cambridge

32. San Andres LA, Vance JM (1987) Effects of fluid inertia on finitelength squeeze-film dampers. ASLE Trans 30:384-393. https:// doi.org/10.1080/05698198708981771

33. Hamzehlouia S, Behdinan K (2016) First order perturbation technique for squeeze film dampers executing small amplitude 
circular centered orbits with aero-engine application. In: Volume 4B: Dynamics, vibration, and control. ASME, p V04BT05A064

34. Hamzehlouia S, Behdinan K (2017) A study of lubricant inertia effects for squeeze film dampers incorporated into high-speed turbomachinery. Lubricants 5:43. https://doi.org/10.3390/lubri cants5040043

Publisher's Note Springer Nature remains neutral with regard to jurisdictional claims in published maps and institutional affiliations. 